\title{
The Quality Chain Management: A Framework for the Prevention and Countermeasures against Non-traditional Security Threats in Xinjiang
}

\author{
Liang Xiong ${ }^{1,2, a}$, Weiwei Wang ${ }^{2, b}$, Silan $\mathrm{Li}^{2, \mathrm{c}}$ and $\mathrm{Xin} \mathrm{Xu}^{2, \mathrm{~d}}$ \\ ${ }^{1}$ Social Science Academy of Xinjiang, Urumqi 830001, China; \\ ${ }^{2}$ Military Economics Academy, Wuhan 43005, China. \\ amilitaryx|@163.com, b35582368@qq.com, cyvonne_1071@126.com and dxuxin@163.com
}

\begin{abstract}
The prevention and countermeasures against non-traditional security threats in Xinjiang (henceforth, the PCNSTX for short) should be viewed as a tough and urgent task that needs systematic organization and long-term efforts. The correlation of life cycle phases of non-traditional security threats in Xinjiang show the chain structure of prevention and countermeasures in each phase, which reveals that quality chain management is an effective way to obtain better PCNSTX. The implementation framework of quality chain management of the PCNSTX consists of 4 levels, that is, the foundation level, technology level, execution level and aim level. Its core is to strengthen the PCNSTX and its ultimate goal is to realize the continuous improvement of the PCNSTX.
\end{abstract}

Keywords: The quality chain management; non-traditional security; prevention; countermeasures; framework.

\section{Introduction}

Compared with the non-traditional security issues in other provinces in China, the non-traditional security threats in Xinjiang are more comprehensive, complex, diverse and uncertain. In this sense, the prevention and countermeasures against non-traditional security threats in Xinjiang (henceforth, PCNSTX for short) should be viewed as a tough and urgent task that needs systematic organization and long-term efforts. In order to better carry out the prevention and countermeasures, a systematic and rational framework is required. This paper aims at creating a framework of the PCNSTX, which is based on the quality chain management.

\section{The Description of Problem}

The PCNSTX should run through all the phases of the whole process. Or rather, the prevention and countermeasures should cover all the phases, that is, the hidden phase, the sign phase, outbreak phase, mitigation phase and diminishing phase. We carry out check, early warning, intervention, control and removal of threats, so as to minimize the detriment caused by non-traditional security threats. In terms of implementation, the aforementioned 5 phases focus on eliminating the effects resulting in the non-traditional security threats in Xinjiang. In these 5 phases, every effort is supposed to be made to find out the effective countermeasures to make the factors that are prone to the occurrence of non-traditional security threats in Xinjiang isolated.

In the PCNSTX, the outcome of the former phase will have direct impact on the latter phase. There is a causal relationship among them and they are interlinked and follow a particular sequence. As it is indicated in chart 1 , these 5 different phases are closely related and dependent on each other. 


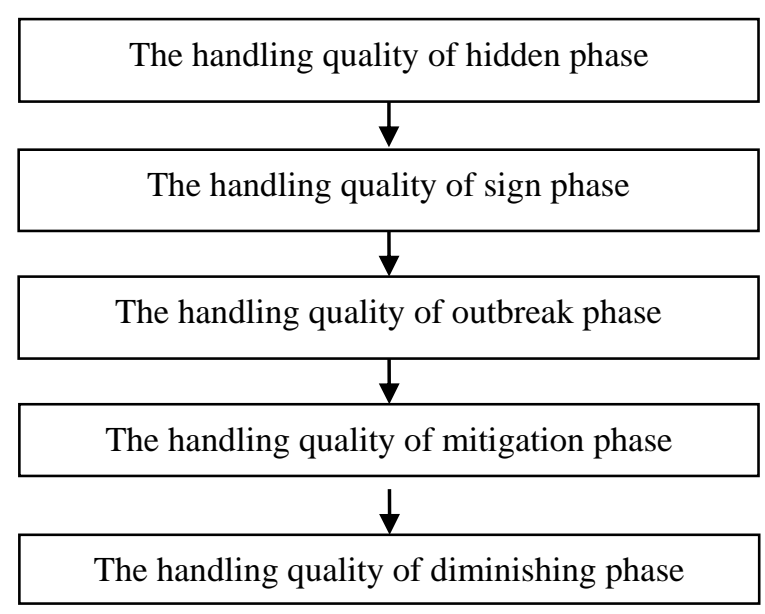

Fig.1 The Quality Chain Management of the PCNSTX

It can be easily figured out that the quality of the PCNSTX dependents on the accumulated effects/outcomes of the 5 phases (the hidden phase, the sign phase, outbreak phase, mitigation phase and diminishing phase). If in each phase, everything is properly handled, finally the prevention of non-traditional security threats in Xinjiang will be strengthened and the quality of countermeasures will be improved.

As is indicated in chart 2, the first piece of dominoes represents the handling quality of the hidden phase, and in this order the last piece of dominoes represents the quality of prevention of non-traditional security threats in Xinjiang. According to the domino theory, if one falls, it knocks down the next one, which will knock down the next one and so on. In this sense, if the handing quality of hidden phase is poor (the first piece representing the handling quality of the hidden phase falls), it will produce an impact on the following pieces and result in the unsatisfied outcome of the non-traditional threats in Xinjiang. Similarly, if the in the hidden phase, everything is properly handled, reaching the required standard, while any other part on the chain, that is, the sign phase, outbreak phase, mitigation phase or diminishing phase, found to have problems unsolved (the piece representing it falls), it will knock down the last piece which represents the quality of prevention of non-traditional security threats in Xinjiang.

If we want to achieve a better prevention of non-traditional security threats in Xinjiang and work out optimum countermeasures, strengthening the handling quality in each phase will be crucial. The correlation of each phase in the life cycle of non-traditional security threats in Xinjiang reveal its chain structure, which also determines that quality chain management is an undoubted choice for improving the PCNSTX.

\section{Model Building}

\subsection{The Connotation of Quality Chain Management of the PCNSTX.}

It is a kind of quality management pattern with chain structure, and the core of this pattern is the quality of the PCNSTX.

First, the scope of quality chain management of the PCNSTX refers to the whole process, which is related to all the phases concerning the PCNSTX, including the personnel, funds, materials and information required in each phase as well as the preparation, early-warning, response, removal of threats and the handling of the aftermath, which all belong to the category of quality chain management. It is a kind of embodiment of whole process quality management of the PCNSTX.

Second, the object of quality chain management of the PCNSTX refers to all the personnel related to the PCNSTX, that is, from personnel engaged in the prevention work in the hidden phase at the very beginning to the personnel in charge of the last part in dealing with the aftermath. And everyone 
participating in the whole process of the prevention and working out the countermeasures should be included in the chain and be regarded as the one of the objects.

Third, the content of quality chain management is to define the responsibility of the PCNSTX. The chain structure of quality management of the PCNSTX is complex and if we want to eliminate the adverse effects to the maximum, this chain or this system should be well-organized, and we have to make the quality management both feasible and successful. In order to achieve this, to define the individual's responsibilities, or the division of labor, becomes very crucial. Every one engaged in the process concerning the quality should be aware of their own responsibilities

Fourth, the aim of quality chain management of the PCNSTX is to achieve the continuous improvement of the PCNSTX. The quality of the PCNSTX will have an impact on maintaining the security and long-term peace and order of Xinjiang.If we carry out quality chain management and pay attention to management of each part on the chain, it will definitely promote the sustainable improvement of the prevention and handling quality.

\subsection{The Basic Framework of the Quality Chain Management of the PCNSTX.}

Due to the differences in function, interests, quality culture and the scattered distribution, each member on the quality chain probably cannot reach a consensus sometimes, which renders the quality chain management of the PCNSTX a complex system, involving several aspects such as technology and management. In order to make it function well, all members' involvements as well as the feasible framework (for the technology and management) for the implementation of quality chain management are required. Now let us suppose that the implementation framework of quality chain management of the PCNSTX consists of 4 levels, that is, foundation, technology, execution and aim, which are shown in the chart 3.

The technology level, including comprehensive management mechanism, information sharing mechanism and collaborative work mechanism, is not only the core for the implementation of quality chain of the PCNSTX, but also the technology platform used for carrying out the quality chain management of the PCNSTX. The comprehensive management mechanism consists of the assessment and appraisal of the quality system of members on the quality chain, the supervisory control of the fluctuation and abnormal performance of quality chain; the information sharing mechanism helps all the members within the quality chain share the information; the collaborative work mechanism is of great help to members on the quality chain in exchanging opinions with each other and working together to solve the problems so as to improve the PCNSTX.

The execution level, between the foundation level and technology level, shows the process of the quality chain management of the PCNSTX. It aims at establishing an agile and efficient quality chain system consisting of organization, management, process and information through the smooth quality flow information.

The aim level, obviously, it is the aim that the quality chain framework is supposed to achieve. The ultimate aim of the quality chain management of the PCNSTX is to realize the smooth, quick, continuous and stable quality flow which includes the quality information flow, design quality flow, demand feedback flow, quality plan flow, quality improvement flow and quality control flow, etc. 


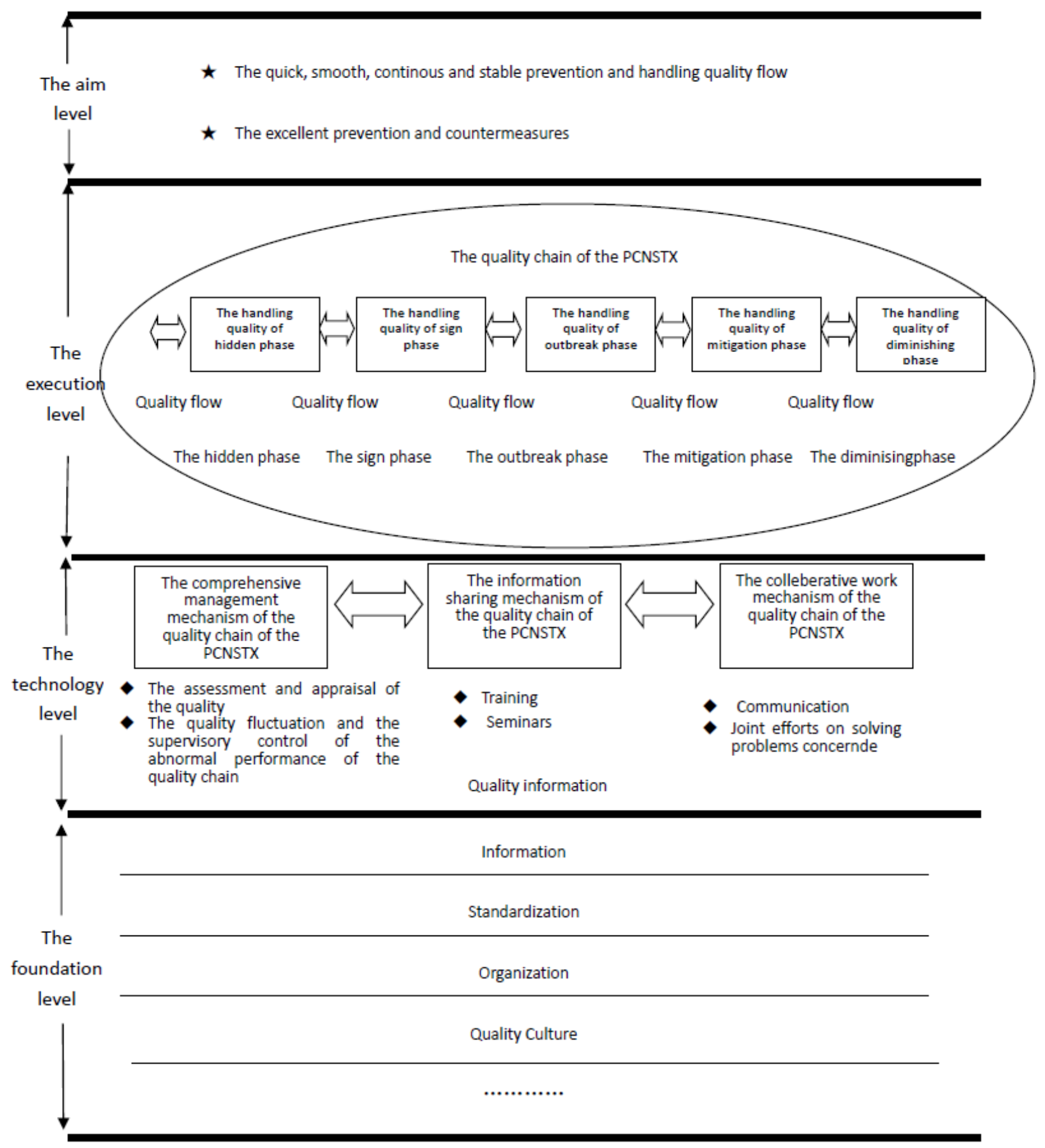

Fig.2 A Framework of Quality Chain Management for the PCNSTX

\subsection{The Key Points to carry out the Quality Chain Management of the PCNSTX.}

The quality chain management framework has been applied in the enterprises management and it works well, and it is worthy of applying quality chain management framework in the PCNSTX.

First, we need to probe into the research of the key technology which is closely related to the quality chain management of the PCNSTX. The quality chain management of the PCNSTX is an advanced management concept incorporating many aspects, such as technology and management. And what's more, various kinds of sophisticated technology are applied. In this sense, it is a must for us to make a deep analysis about the key technologies used for quality chain management. The coordination service technology is such an important technology that is required in this management. The PCNSTX is very complex and people will be more and more strict about the implementation of the PCNSTX. And when put into practice, both the prevention and handling measures and each phase on the chain (life cycle) will become complicated. And if we want to obtain a better outcome, every member on the quality chain needs to work with coordination and by doing this it is likely to 
accomplish the task perfectly. Besides, the appraisal technology of each member on the quality chain is also required. In the whole process of the PCNSTX, a large number of departments and personnel will be involved in and the decision they make and actions or activity they carry out have direct impact on the outcome of the PCNSTX. In this sense, we need to strengthen the control of each member on the quality chain and make accurate assessment and appraisal on the performance of members. Apart from these two technologies, the process modeling technology is also a must. The quality chain of the PCNSTX is composed by a series of processes concerning quality and it is complex and dynamic. In order to better understand the operation of the whole process and strengthen the control of it, we need to build a model to guide its operation. At present, the method for building model used by the enterprises can be adopted, such as the method based on rule, process programming method and the method based on function.

Second, we need to pay much attention to the quality culture development of each member on the quality chain. In 1993 the World Conference on Quality and International Academy for Quality reached a consensus that the quality management was no longer the pursuit of total quality management and the enterprise quality culture became the focus. This just indicates that the quality culture is now the core of enterprise culture of quality chain member. And when we introduce the quality chain management, a kind of work needs to be done first, that is, enhancing the quality culture development of the PCNSTX. The quality culture here refers to a kind of value, aim and belief which can be found in the process of pursuing the best products in an activity in which people are supposed to exert themselves in pursuing high quality. And for all the members on quality chain of the PCNSTX, they are required to transform the quality value into the working style so as to improve their work and finally achieve the aim (the PCNSTX of high quality).Because of the significance of quality culture, on the one hand, we need to make it clear that the quality culture has a special status. Once the excellent quality culture takes shape, it will have profound impact on all the members on the quality chain of the PCNSTX. The quality culture and iceberg are similar to some extent, we cannot get the full view of the iceberg in that main body is hidden under the sea, and just because of that the iceberg has enormous power to make it hardly shakable. In the quality chain of the PCNSTX, people's influence on the quality always exists and if we don't make quality culture become a solid foundation, then it is hard for us to guarantee the quality. On the other hand, we need to develop the quality values. The quality values should be thoroughly introduced to all the members on the quality chain so that they will be fully aware of their responsibilities and understand the significance and status of quality in the PCNSTX. We also hope that every member is aware of being responsible for the PCNSTX, willing to pay much attention to the quality control and both active and creative. The training are required to make the personnel concerned grasp the skills and methods, learn to make self-check, self-control and finally make them improved. And it is consistent with the concept that we obtain the high quality relying on the whole-process quality control instead of the quality inspection. In this way members on the quality of the PCNSTX can distinguish themselves from other quality cultures.

\section{Conclusion}

The non-traditional security threats can have major impact on social security, economic security, environmental security, cultural security and peripheral security. From the perspective of life cycle, although the type of the threats may be different from each other, they follow the same pattern, that is, the hidden phase, the sign phase, outbreak phase, mitigation phase and diminishing phase. The preventions and countermeasures should be carried out through the whole process from the very beginning of the hidden phase to the end of the last phase. We need to find out the effective measures and methods to eliminate the factors closely related to the happening, development of the non-traditional security threats as much as possible. There is a causal relationship among the outcome of each phase and they are interlinked and follow a particular sequence and form a kind of dominoes.

The correlation among phases of life cycle of the non-traditional security in Xinjiang implies its chain structure, and the implementation of the quality chain management shall be the effective way to 
improve the prevention and countermeasures. The framework of the PCNSTX is established based on the quality chain management and its core is to strengthen the PCNSTX and the ultimate goal is to realize the improvement of prevention and countermeasures. The implementation framework of quality chain management of the PCNSTX consists of 4 levels, that is, foundation, technology, execution and aim. Its scope is the whole process of coping with the problems concerning the non-traditional security threats in Xinjiang. All the personnel involved in the PCNSTX are under the management of the quality chain. The content of this management is to define the responsibilities of the PCNSTX and the aim of the management is to realize the continuous improvement of the PCNSTX.

\section{References}

[1] Xiaoping An,Guiping Xie, Xinming,Li. On Special Characteristics of Non-traditional Security Threats in Xinjiang, Journal of Tarim University,2010 (3)

[2] Yuezhen Liu. Study on Challenges and Countermeasures of Non-traditional Security Threats in Xinjiang. Economy and Culture of the Frontier, 2009(8)

[3] Hangong Wang, Maozhi Gan. The Whole-life-span Management of the Equipment, National Defense Industry Press, Beijing,2003

[4] Quanxi Li, Xin Zhang, Jiao Wang. The Quality Chain Management in the Mode of Advanced Manufacturing. The Science and the Scientific Technology Management, 2004(7)

[5] Xiaoqing Tang, Guijiang Duan. The Coordinative Quality Chain Management in the Globalized Manufacturing. China Quality, 2002(09)

[6] Yijun Ren. A Tentative Study on the Higher Education Quality Chain, Heilongjiang Higer Education Research, 2001(06)

[7] Shucheng Ma, Xiaoqing Tang.The Features and Strategy of Supply Chain Quality Management, The Computer Integrated Manufacturing System, 2001(9)

[8] Wenqin Ding, Junhua Zhao. Quality Chain-Quality Dominoes-How to Improve the Quality of Products, The Chemical Industry Quality, 1999(6) 